\title{
Superior ophthalmic vein thrombosis associated with orbital inflammation
}

\begin{abstract}
We report a case of a 71 year old female who presented with redness, pain and periocular swelling in the left eye of 3 months duration and decreased vision and protrusion since 15 days. Previous ophthalmological history was unremarkable. The vision was PL negative. Examination showed moderate axial proptosis, mild ptosis, fullness of the eyelids, and a chemosed, prolapsed conjunctiva. Fundus examination showed a branch vein occlusion of the inferotemporal vein. There was total ophthalmoplegia OS. A provisional diagnosis of pseudotumour was made after basic investigations, and steroid therapy was initiated, but the patient showed little response. CT orbit showed left eye proptosis with minimal fat stranding and superior ophthalmic vein thrombosis which was confirmed by MRI. She also had an intracranial infarct which was picked up on MRI. Due to multiple vaso occlusive sites, and the lack of response to steroid therapy, she was referred for a haematological work up. Superior ophthalmic vein thrombosis, although rare, can be a harbinger of cavernous sinus thrombosis, hence the need for an urgent and detailed evaluation of orbital thrombosis.
\end{abstract}

Volume 7 Issue 2 - 2017

\section{Bhargavi Pawar,Ann Mary Matthews}

Department of Ophthalmology, St John's Medical College, India

Correspondence: Bhargavi Pawar, Assistant Professor of Ophthalmology, St John's Medical College, Sarjapur Road, Bangalore 560034, India, Tel +9l-9449833078,

Email dr.bhargavipawar@gmail.com

Received: June 30, 2017| Published: July 18, 2017

Keywords: superior ophthalmic vein thrombosis, ophthalmoplegia, orbital inflammation

\section{Introduction}

Superior ophthalmic vein thrombosis (SOVT) is commonly associated with orbital inflammation and congestion such as orbital cellulitis, idiopathic orbital inflammation, vascular malformations, thyroid-related orbitopathy, trauma and orbital tumors. ${ }^{1-3}$ The clinical signs may resemble those of orbital conditions such as proptosis, ophthalmoplegia and loss of visual function. Radiological tests like MRI are sensitive and can detect a thickened and dilated Superior Ophthalmic Vein (SOV) in the early stage of disease. The treatment lies in identifying the underlying cause and treating accordingly. SOV could be life threatening since it holds an impending risk of cavernous sinus thrombosis. ${ }^{1-4}$

\section{Case discussion}

A 71 year old female who presented with redness, pain and periocular swelling of the left eye (OS) since 3 months, and decreased vision and protrusion OS since 15 days. She had been referred from the neurology department for an ophthalmological opinion. The patient was a diabetic and hypertensive since 2 years, on treatment. Her BCVA was 20/80 OD and PL negative OS. Right eye examination was normal except for a senile immature cataract. Examination of the left eye showed moderate axial proptosis, mild ptosis, fullness of the eyelids, and a chemosed, prolapsed conjunctiva (Figure 1,2). There was diffuse congestion of the ocular surface and some tortuous episcleral vessels. The corneal surface had multilple punctate erosions which was attributable to the proptosis. The pupil was $6 \mathrm{~mm}$ and non reactive. A hazy fundus view showed dilated and tortuous veins with haemorrhages along the inferotemporal arcade suggestive of a branch vein occlusion. There was total ophthalmoplegia OS (Figure 3). The globe was tense and there was resistance to retropulsion. Intraocular pressure was $28 \mathrm{~mm} \mathrm{Hg}$. There was no palpable thrill or bruit heard.

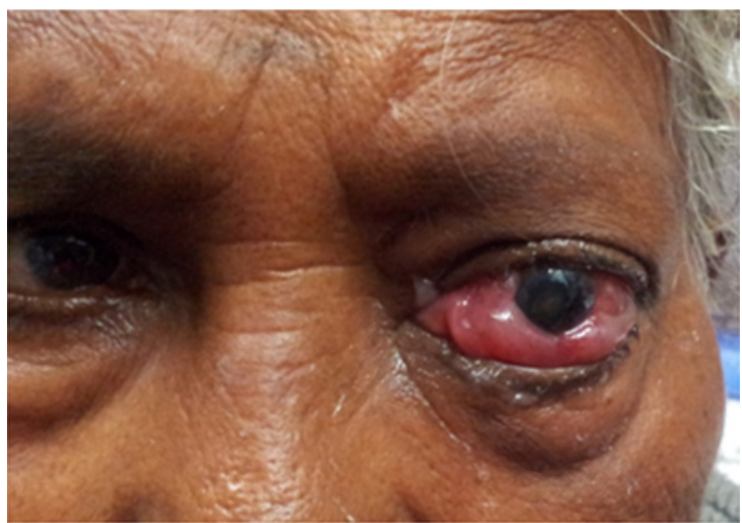

Figure I Moderate proptosis OS, with prolapsed and chemosed conjunctiva

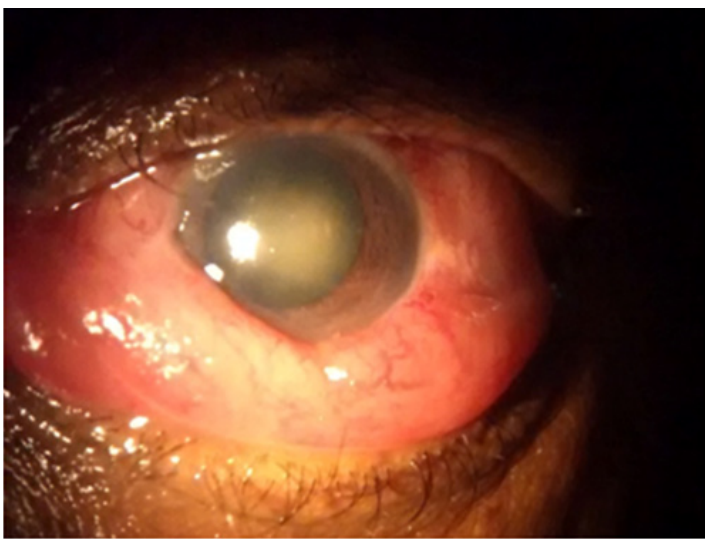

Figure 2 Chemosis, congestion and fixed dilated pupil, suggesting compromised optic nerve function. 


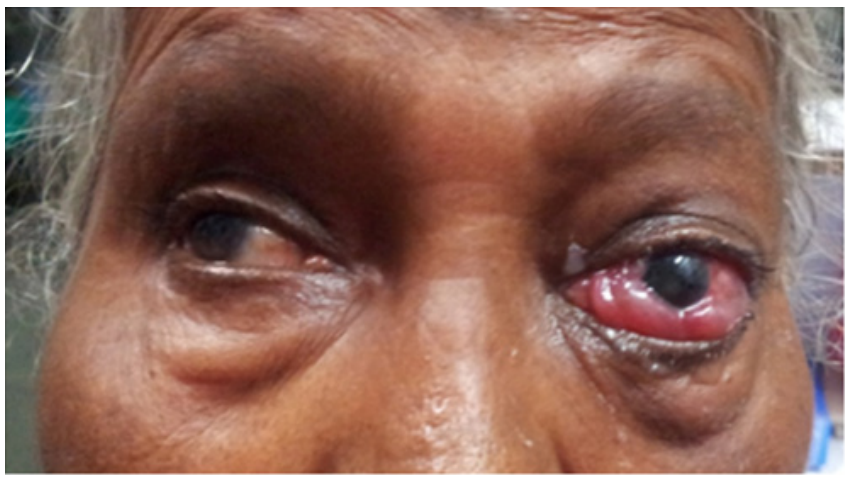

Figure 3 Restricted ocular motility OS as seen in dextroelevation.

Blood counts and investigations were as follows: $\mathrm{Hb} 11.6 \mathrm{~g} \%$, total count 9300 with neutrophil $58.4 \%$, lymphocytes $29 \%$, random sugar levels were fluctuating, Blood urea 19, serum creatinine 0.69. Prothrombin time of 11.3s and INR 1.01.Mantoux test was negative for tuberculosis. Nasal swabs and culture ruled out bacterial and fungal sinusitis. Thyroid profile was within normal limits, Anti neutrophil cytoplasmic antibody (ANCA) was negative, and Angiotensin converting enzyme (ACE) levels were within the normal range, hence we were able to rule out thyroid orbitopathy, sarcoidosis and wegeners granulomatosis. CT orbit showed left eye proptosis with minimal fat stranding and superior ophthalmic vein enlargement. MRI confirmed a left dilated and engorged SOV, suggestive of SOV thrombosis, congested extraocular muscles and features suggestive of compressive optic neuropathy (Figure 4). The MRI also identified an acute ischemic infarct with haemorrhagic transformation in the left lateral aspect of pons in the brain.

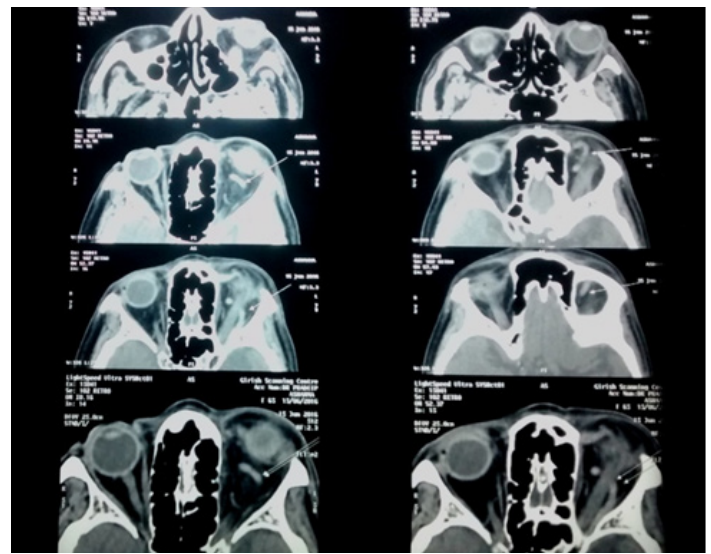

Figure $4 \mathrm{MRI}$ showing dilated and tortuous superior ophthalmic vein in multiple sections (white thin arrows).

A CT/MR angiography was also ordered to look for the possibility of a low flow carotico-cavernous fistula, but the patient refused the procedure. A provisional diagnosis of idiopathic orbital inflammation with SOV occlusion was made. The patient was given a trial of oral prednisolone $1 \mathrm{mg} / \mathrm{KGB}$ for 72 hours but there was no response to the steroid therapy nor was there an alleviation of symptoms. In view of the multiple vaso-occlusive sites - intraocular, intraorbital and intracranial, a referral to the physician and neurologist for a complete haematological work up and initiation of anticoagulant measures was made. The patient was transferred to the neurology department for management of the intracranial vascular occlusion and was thereafter lost to follow up.

\section{Discussion}

In patients with orbital infection, the concurrent orbital congestion can incite an inflammatory response that results in thrombophlebitis and thrombus formation; alternatively, thrombosis may be caused by direct vascular invasion by the pathogen. ${ }^{1}$

\section{Clinical presentation}

Patients with SOVT may present with orbital or facial pain and swelling, diplopia, or decreased vision. Clinical findings may include proptosis, chemosis, ophthalmoplegia, and ptosis. The optic nerve may be affected by compression, with clinical signs of optic neuropathy such as reduced visual acuity, abnormal color vision, and relative afferent pupillary defect and could warrant imaging and surgical intervention. ${ }^{1,4}$

\section{Risk factors}

The risk factors for SOV thrombosis include result of infection, trauma, inflammation, hypercoagulable states, neoplasm, and orbital crowding.

\section{Imaging}

In the early stages, a mildly enlarged SOVT may be missed on CT scans, and decreased blood flow through the SOV and cavernous sinus may not be apparent. Magnetic resonance imaging (MRI) and magnetic resonance venography are more sensitive early in the disease process and are recommended if there is suspicion of SOVT or CST. ${ }^{1,4}$ It may reveal a dilated SOV or congested muscle.

\section{Diagnostic evaluation}

In view of the possible systemic associations of orbital inflammation, inflammatory and autoimmune workup may be indicated, including laboratory tests for serum angiotensin-converting enzyme, rheumatoid factor, antineutrophil cytoplasmic antibody, anti microsomal antibody, and thyroid function. Chest $\mathrm{x}$-ray is indicated to evaluate for sarcoidosis. Patients with SOVT should be evaluated, in consultation with a physician or internist for underlying systemic illness. Laboratory investigation should include a complete blood count looking for leukocytosis or left shift, an increase in the ratio of immature to mature leukocytes. If there are no signs of infection or inflammation, evaluation for a hypercoagulable condition may be indicated, including factor V Leiden, prothrombin gene mutation, lupus anticoagulant, and antithrombin III. ${ }^{1,5}$

\section{Management}

The appropriate management of SOVT depends on the etiology. It may be infectious, inflammatory or coagulopathies. In case the etiology is found to be infectious, broad spectrum intravenous antibiotics are recommended and should be continued for up to 2 weeks if beyond clinical resolution of the infection. Remote sources of infective emboli such as bacterial endocarditis should also be explored. The role of anticoagulation therapy in isolated SOVT is unclear and is considered after risk benefit analysis. ${ }^{5}$ However, SOVT that is not treated with anticoagulation may progress to cavernous sinus thrombosis and if there is no contraindication, anti coagulant therapy can be initiated by the physician/ internist.

Dose-adjusted intravenous (IV) heparin is the recommended treatment. One randomized controlled trial suggests an IV bolus injection of 3,000 IU followed by 25,000 to $65,000 \mathrm{IU} /$ day continuous 
IV infusion, adjusted according to prothrombin time (PTT) such that the PTT is doubled but does not exceed 120 seconds. ${ }^{1,6}$ Corticosteroid therapy may help limit the inflammation in thrombophlebitis, but is beneficial largely in idiopathic orbital inflammatory disease. It has been suggested that noninfectious SOVT may be a variant of idiopathic orbital inflammatory disease, which may be steroid responsive. ${ }^{1}$ From our case study, we would like to recommend that, in an idiopathic orbital inflammation, if the patient does not respond to high dose steroid therapy within 72 hours, there is an urgent need to explore systemic etiological factors.

\section{Conclusion}

A presumed idiopathic orbital inflammatory disease needs further evaluation if there is no response to high dose steroid therapy. A haematological work up of systemic autoimmune diseases as enumerated is essential to the management of SOV occlusion. SOVT could be an early sign of impending cavernous sinus thrombosis which could be life threatening. ${ }^{1,4}$ Anticoagulants may be considered in such patients, after weighing the risks and benefits, in consultation with the treating internist. A multidisciplinary approach with the treating physician, neurologist, radiologist and ophthalmologist is suggested when managing a case of orbital vein thrombosis. MRI and MRV is the imaging modality of choice for confirming SOVT and can help to exclude other disorders which may mimic the condition. ${ }^{1,5,7}$

\section{Acknowledgments}

None.

\section{Conflicts of interest}

The authors declare no conflicts of interest.

\section{References}

1. Jaya BK, Betsy CA, Jason L, et al. Diagnosis and Management of Superior Ophthalmic Vein Thrombosis. Eye net magazine. 2015.

2. Vyas S, Das PJ, Gupta SK, et al. Ophthalmic Veins Thrombosis with Cavernous Sinus Meningioma. Middle East Afr J Ophthalmol. 2011;18(3):256-258.

3. Grassi MA, Lee AG, Kardon R, et al. A lot of clot. Surv Ophthalmol. 2003;48(5):555-561.

4. Berenholz L, Kessler A, Shlomkovitz N, et al. Superior ophthalmic vein thrombosis: Complication of ethmoidalrhinosinusitis. Arch Otolaryngol Head Neck Surg. 1998;124(1):95-97.

5. Lim LH, Scawn RL, Whipple KM, et al. Spontaneous superior ophthalmic vein thrombosis: a rare entity withpotentially devastating consequences. Eye. 2014;28(3):348-351.

6. Einhäupl KM, Villringer A, Meister W, et al. Heparin treatment in sinus venous thrombosis. Lancet. 1991;338(8767):597-600.

7. Karanbir Singh, Deeya Gaindh, Ghulam Mustafa, et al. Spontaneous superior ophthalmic vein thrombosis: a case report (P6.227). Neurology 84(14 Supplement P6): 227. 